\title{
Geoquímica de lantánidos de los yacimientos de fluorita de los distritos mineros de Taxco y Zacualpan, sur de México: implicaciones sobre el origen y la evolución de los fluidos
}

\author{
Teresa Pi ${ }^{1,2 *}$, Jesús Solé ${ }^{1,2}$, Ofelia Morton-Bermea ${ }^{3}$, Yuri Taran ${ }^{3}$ y Elisabeth Hernández-Álvarez ${ }^{3}$ \\ ${ }^{1}$ Instituto de Geología, Universidad Nacional Autónoma de México, Col. UNAM C.U, Coyoacán, 04510, Cd. de México, México. \\ ${ }^{2}$ Laboratorio Nacional de Geoquímica y Mineralogía (LANGEM), Universidad Nacional Autónoma de México, \\ Col. UNAM C.U., Coyoacán, 04510, Cd. de México, México. \\ ${ }^{3}$ Instituto de Geofísica, Universidad Nacional Autónoma de México, Col. UNAM C.U., Coyoacán, 04510, Cd. de México, México. \\ *tpuig@geologia.unam.mx
}

\section{RESUMEN}

Se presentan y evalúan los contenidos de lantánidos, analizados mediante espectrometría de masas de plasma inducido (ICP-MS), en fluoritas de diferentes yacimientos de los distritos mineros de Taxco y Zacualpan situados en el sector sur de México. Esta información es utilizada como herramienta para establecer las relaciones de este mineral con la roca encajonante, así como para discriminar diferentes episodios de mineralización y la existencia de procesos postdeposicionales.

Las fluoritas analizadas presentan contenidos variables en lantánidos y de manera predominante patrones normalizados enriquecidos en lantánidos ligeros (LREE). La concentración de lantánidos en la fluorita es baja en comparación con la que presentan las rocas volcánicas y metamórficas del encajonante ( $\mathrm{REE}>100 \mathrm{ppm})$ y alta respecto a la de los carbonatos ( $\mathrm{REE}<30 \mathrm{ppm})$. Existe una correlación entre la forma de los patrones de lantánidos de la fluorita y la naturaleza de la roca huésped. Las concentraciones más altas se dan en fluorita hospedada en rocas volcánicas y las más bajas en la fluorita asociada al reemplazo del carbonato. Por su parte, la fluorita asociada a sulfuros presenta un mayor enriquecimiento en lantánidos pesados. Casi todas las fluoritas presentan anomalía negativa de europio con excepción de algunas fluoritas tempranas de color oscuro y alto contenido de uranio de la mina La Azul. En algunas muestras se observa una correlación directa entre el color y el contenido en lantánidos.

Integrando todos los datos, en el distrito minero de Zacualpan se ha podido discriminar un solo episodio de mineralización. La fluorita presenta características geoquímicas típicas de las fluoritas hidrotermales.

En el distrito minero de Taxco, y particularmente en la Mina La Azul, se han diferenciado varias etapas de mineralización. La primera generación de fluorita está asociada al reemplazo de carbonatos y se caracteriza por un contenido muy bajo de lantánidos, y patrones de lantánidos normalizados similares a los de las rocas carbonatadas, alto contenido de estroncio y texturas primarias (p. ej., fluorita masiva y ritmitas). La segunda generación de fluorita está relacionada con la entrada de nuevos fluidos al sistema y se caracteriza por presentar concentraciones más altas de lantánidos, patrones similares a los de las rocas volcánicas, bajo contenido de estroncio y texturas secundarias (p. ej., brechas y nódulos). Por lo que respecta a su génesis, los patrones de lantánidos de la mayoría de las muestras de fluorita del distrito minero de Taxco evidencian una relación directa con fluidos de origen magmático.

Palabras clave: fluorita; lantánidos; REE; hidrotermal.

\begin{abstract}
We present and evaluate lanthanide contents measured by inductively coupled plasma mass spectrometry (ICP-MS) in fluorite samples from the fluorite deposits in Zacualpan and Taxco mining districts in the south of Mexico. The information is used to distinguish different generations of fluorite, to establish a correlation between mineralization episodes and the wall rock nature, and to identify postdepositional processes.

The total lanthanide content of the fluorites are variable, and earlystage fluorite samples are usually enriched in LREE. The concentration of $R E E$ in fluorite is low in comparison with the volcanic and metamorphic rocks ( $\Sigma R E E>100 \mathrm{ppm})$ and is generally high respect the carbonates $\left(\sum R E E<30 \mathrm{ppm}\right)$. There is host rock influence. The higher REE concentrations are in fluorites hosted by volcanic rocks. The fluorite that replaced carbonate is characterized by low REE to very low concentrations. Fluorite samples associated with sulfurs are typically enriched in HREE. Nearly all fluorites show a negative Eu anomaly similar to the REE anomaly observed in the volcanic rock. Only some early stage dark, uranium rich fluorites, from la Azul deposit, have a strong positive Eu anomaly. Direct correlation between color and REE patterns is observed in some samples.

In the Zacualpan mining district, only an episode of mineralization has been discriminated, where fluorite presents flat to HREE- enriched chondrite-normalized REE patterns.

In the Taxco mining district and particularly in the "Mina la Azul", multiple hydrothermal events of mineralization have been determined. The first generation of fluorite is formed by replacement of carbonates and is characterized by very low contents of lanthanides, chondrite-
\end{abstract}


normalized REE patterns similar to the limestone, high strontium content and primary textures (e.g. massive fluorite and rhythmites). The second generation of fluorite is related to the entry of new fluid to the system and has higher REE concentrations, chondrite-normalized REE patterns similar to volcanic rocks, low strontium content and secondary textures (i.e. breccias, nodules). Most of the samples show a genetic relationship between fluorite and fluids of magmatic origin.

Key words: fluorite; lanthanide; REE; hydrothermal.

\section{INTRODUCCIÓN}

En los sistemas hidrotermales la distribución de lantánidos está controlada por la partición de estos elementos entre las soluciones y las fases sólidas que precipitan y por la interacción del fluido hidrotermal con la roca hospedante. Desde hace décadas la geoquímica de lantánidos se ha convertido en una herramienta muy útil en el estudio de algunos tipos de mineralizaciones hidrotermales (p. ej., Wood, 1990; Monecke et al., 2000; William-Jones et al., 2000; Sasmaz y Yavuz, 2007; Ehya, 2012). El principal problema es que casi todos los datos experimentales de los coeficientes de partición de lantánidos en minerales se refieren a sistemas magmáticos (p. ej., Irving, 1978), es decir, a sistemas de alta temperatura y presión y no a sistemas que se forman en condiciones más superficiales. Por otra parte, como muchos sulfuros y óxidos no son buenos huéspedes para los lantánidos (Lottermoser, 1992), los estudios sobre la distribución de estos elementos en yacimientos hidrotermales se limitan a los minerales de ganga como son la calcita, la fluorita y la barita.

El comportamiento geoquímico de los lantánidos en sistemas hidrotermales es complejo, ya que está influenciado por casi todos los parámetros y procesos que intervienen en la formación de los depósitos: temperatura, presión, $\mathrm{pH}$, Eh e interacción roca-fluido (p. ej., Lottermoser, 1992). La variación de uno de estos parámetros, puede producir una desestabilización de los complejos químicos que transportan a los lantánidos y determinar a su vez modificaciones importantes en los patrones. En los minerales hidrotermales la distribución de lantánidos dependerá del mineral analizado y de la posición temporal del mineral en la secuencia paragenética. Al precipitar un mineral se puede producir un fraccionamiento que afectará principalmente a la concentración de Eu (anomalías positivas y negativas) y a la relación de lantánidos ligeros respecto los lantánidos pesados.

Mediante espectrometría de masas con fuente de plasma de acoplamiento inductivo (ICP-MS) se han determinado los contenidos en lantánidos de fluorita de diferentes yacimientos de los distritos de Taxco (Mina La Azul, Mina Don Baldomero, Mina Los Tréboles, Mina el Gavilán) y Zacualpan (Mina La Natalia, Mina el Perpetuo Socorro y Mina General Escobedo) situados en el sector sur de México con la finalidad de correlacionar estos patrones de lantánidos con las rocas encajonantes, reconstruir la evolución del fluido mineralizante y revisar si existe una correlación clara entre el color y el contenido de lantánidos de las fluoritas. La selección de muestras se realizó con base en los estudios mineralógicos, geoquímicos y geocronológicos previos realizados en un total de 220 muestras (Pi, 2005 y Pi et al., 2005a, 2005b).

\section{ANTECEDENTES}

Fleischer (1969) reportó variaciones considerables en la composición de lantánidos de fluoritas hidrotermales, incluso dentro de un mismo depósito. Más recientemente la geoquímica de lantánidos en fluoritas ha sido utilizada por muchos autores como indicadora del tipo y del origen de los fluidos asociados (p. ej., Grappin y Treuil, 1979; Ruiz et al., 1980; Chatagnon y Galland, 1982; Strong et al., 1984; Jebrack et al.,1985; Ekambaram et al., 1986; Constantopoulus, 1988; Eppinger y Closs, 1990; Subías et al.,1994; Bau y Dulski, 1995; Fanlo et al., 1998; Bosze y Rakovan, 2002; Gagnon et al., 2003; Trinkler et al., 2005; Soussi et al., 2012; Makin et al., 2014). Esta posible utilización como mineral trazador se debe a la facilidad que presentan los átomos de Ca de la fluorita $\left(\mathrm{CaF}_{2}\right)$ de ser sustituidos por cationes de igual carga y radio iónico parecido.

Posibles aplicaciones de la geoquímica de lantánidos en fluorita

Algunos aspectos particulares que, según diferentes autores, se pueden deducir con base en el estudio de la concentración y distribución de lantánidos en fluorita son:

a) Definir de forma detallada la secuencia paragenética de un depósito: si no hay un fraccionamiento de lantánidos durante la cristalización de la fluorita se pueden discriminar los estadios de mineralización de un depósito (p. ej., Marchand et al., 1976; Möller y Morteani, 1983; Constantopoulus, 1988) y los yacimientos de un mismo distrito minero (p. ej., Schwinn y Markl, 2005).

b) Origen del fluido y relación del mismo con el encajonante: es frecuente que el espectro de lantánidos de la solución sea heredado de la roca alterada (Marchand et al., 1976). La fluorita adquiere la composición de REE de la roca original modificada en función de la naturaleza y características geoquímicas del fluido (p. ej., Hill et al., 2000; Sasmaz y Yavuz, 2007).

c) Definir los procesos asociados a la mineralización: existen diferentes relaciones entre concentraciones de lantánidos para cuantificar el fraccionamiento y determinar cuando éste está asociado a procesos evolutivos del fluido, a remobilización tardía o a la asimilación del encajonante (p. ej., Möller et al., 1976; William-Jones et al., 2000; Xu et al., 2012).

d) Condiciones redox del fluido: las anomalías de Eu y Ce indican las condiciones de fugacidad de oxígeno del fluido a partir del cual precipitó la fluorita (p. ej., Bau et al., 2003).

e) Génesis del mineral: en casos favorables la utilización de las relaciones entre concentraciones de lantánidos permite discriminar entre fluorita de origen neumatolítico, hidrotermal o sedimentario (Möller et al., 1976).

f) Fines exploratorios: discriminar si la fluorita está asociada a sulfuros. Varios autores (p. ej., Eppinger y Closs, 1990; Hill et al., 2000; Graupner et al., 2015) han caracterizado diferentes tipos de yacimientos de fluorita en función del contenido de REE y reportan que esta información puede ser utilizada en la prospección de minerales metálicos.

\section{Procesos de substitución del Ca de la fluorita por elementos traza y anomalía de Eu}

Muchas fluoritas son en un $99 \%$ o más $\mathrm{CaF}_{2}$ con pequeñas cantidades de $\mathrm{Si}, \mathrm{Al}$ y $\mathrm{Mg}$ y en menor proporción $\mathrm{Sr}, \mathrm{Y}, \mathrm{Be}, \mathrm{Ag}, \mathrm{Ba}, \mathrm{Yb}$ y $\mathrm{P}$ (Allen, 1952). Los elementos menores identificados en la fluorita son a menudo encontrados como constituyentes mayores en los minerales asociados ( $\mathrm{Sr}$ en celestita, Ba en barita, etc.).

Los iones de $\mathrm{Na}, \mathrm{Mn}, \mathrm{Fe}, \mathrm{Sr}, \mathrm{Y}, \mathrm{Zr}, \mathrm{Ba}, \mathrm{La}, \mathrm{Eu}, \mathrm{Ce}, \mathrm{Yb}$ y Pb substituyen al Ca en la estructura iónica. Las principales substituciones en fluorita son de Y (hasta 14\%) y Ce (hasta 15\%) y determinan un incremento considerable del tamaño de la celda. Los cálculos teóricos y algunos estudios experimentales sugieren que los lantánidos son incorporados a la estructura cristalina de la fluorita de forma preferente si su radio iónico es próximo al que presentan los posibles espacios a substituir (Morgan, 1980). Según esto, la fluorita incorporaría preferentemente a los cationes entre el $\mathrm{Tb}$ y Ho que tienen un radio iónico inferior al 
$\mathrm{Ca}^{2+}$ (p. ej., Whittaker y Muntus, 1972). Sin embargo, otros autores (p. ej., Meary et al., 1985) demuestran que en ausencia de otros iones que permitan la formación de complejos y siempre que no se produzca la precipitación previa de otras fases minerales, los espectros de lantánidos en fluoritas reflejan los contenidos de la solución de la que derivan.

En soluciones acuosas a $25^{\circ} \mathrm{C}$ el estado dominante de los lantánidos es el trivalente. En condiciones altamente oxidantes se puede formar $\mathrm{Ce}^{4+}$ y en condiciones altamente reductoras $\mathrm{Eu}^{2+}$. Dado que el estado de oxidación del Eu determina su radio iónico $\left(\mathrm{el} \mathrm{Eu}^{2+}\right.$ es $17 \%$ más grande que el $\mathrm{Eu}^{3+}$ ), un cambio en el estado de oxidación puede determinar cambios importantes en los coeficientes de partición de este elemento que se caracteriza por ser el más reactivo de todos los lantánidos. Así, por ejemplo, el $\mathrm{Eu}^{2+}$ substituye más fácilmente que el $\mathrm{Eu}^{3+}$ al Ca y al $\mathrm{Na}$ de la plagioclasa, al Ca de la fluorita o al Ba de la barita determinando anomalías positivas de Eu en estos minerales y anomalías negativas en el magma o líquido residual.

\section{MARCO GEOLÓGICO}

Los depósitos estudiados se ubican en los estados de Guerrero y México y se concentran en dos áreas principales (Figura 1):

a) Al noreste de Taxco cerca de los poblados de Acamixtla y Acuitlapán y cubriendo una distancia aproximada de $10 \mathrm{Km}$. Los

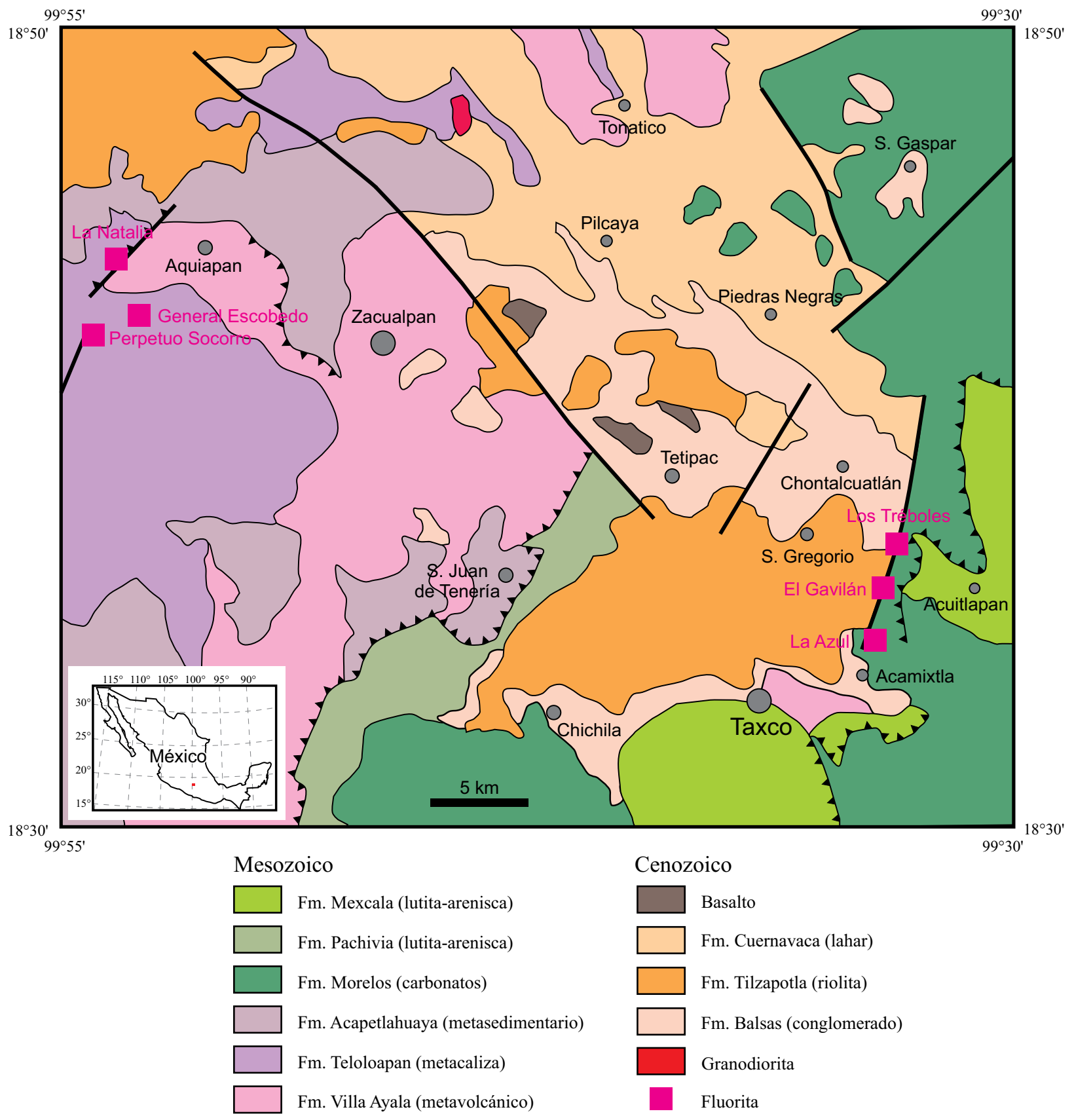

Figura 1. Mapa geológico simplificado con la situación de los principales yacimientos de fluorita estudiados. Simplificado de la hoja Cuernavaca 90_E14-5 (Rivera Carranza et al., 1998). 
yacimientos de fluorita más importantes se encuentran en el contacto tectónico (en la denominada falla de Acamixtla con dirección N10E), entre las rocas volcánicas terciarias y los carbonatos cretácicos. Las rocas volcánicas son principalmente ignimbritas, riolitas y vitrófidos muy ricos en $\mathrm{SiO}_{2}(>67 \%)$, de afinidad calcialcalina y que se relacionan genéticamente con el arco magmático asociado a la subducción del margen Pacífico de México (Alaniz-Álvarez et al., 2002). Estas rocas presentan textura porfídica, matriz vítrea y/o microcristalina y fenocristales de cuarzo, sanidina, plagioclasa y mica. Los carbonatos del Mesozoico constan básicamente de secuencias de plataforma y se relacionan con una transgresión al final del Jurásico. En la zona también aflora una secuencia altamente deformada de rocas volcanosedimentarias de arco magmático metamorfoseadas (carbonatos y detríticos de diferente granulometría) en facies de esquisto verde, de edad cretácica (Campa-Uranga et al., 2012). Como minas principales de fluorita en este distrito destacan la mina La Azul y El Gavilán, como minas de menor importancia Los Tréboles y Don Baldomero. Los cuerpos de fluorita son diversos en morfología y tamaño y la mineralización más importante es la mina La Azul con unas dimensiones aproximadas de $150 \mathrm{~m} \times 200 \mathrm{~m} \times 40 \mathrm{~m}$ de profundidad. Las leyes de fluorita reportadas para estas mineralizaciones son muy variables (8 a $85 \%$ de fluorita).

b) Al poniente de Zacualpan y al suroeste del poblado de Aquiapan. En este distrito minero las mineralizaciones de fluorita se encuentran en vetas hidrotermales encajonadas en secuencias metamórficas de bajo grado (esquistos verdes con sericita y cuarcitas) bastante deformadas aprovechando la presencia de fallas casi verticales y en asociación con vetas de minerales metálicos y vetas tardías de calcita. La secuencia metamórfica en Zacualpan está formada en la base por un paquete complejo de rocas metavolcánicas (basaltos y en menor proporción riolitas y andesitas) calcialcalinas asociadas a arcos de islas intraoceánicos y rocas metasedimentarias en la parte superior. La mina principal es la denominada General Escobedo y otras de menor tamaño son El Perpetuo Socorro y La Natalia. Para este distrito se desconocen las leyes de fluorita de estas mineralizaciones ya que fueron abandonadas hace muchos años.

\section{METODOLOGÍA}

Se analizaron por ICP-MS un total de 81 muestras distribuidas como sigue: 63 análisis de fluorita, dos de barita, siete de carbonatos (sedimentarios e hidrotermales), seis de rocas volcánicas del encajonante y tres de arcillas. En la Tabla 1 se resumen de manera breve las características básicas de las muestras analizadas.

Los análisis se realizaron en dos laboratorios. Una serie se midió en el Instituto de Geofísica de la UNAM (IGF) y otra en Activation Laboratories Ltd (ACTLABS). Se utilizaron dos métodos de disolución que se describen a continuación. Método IGF: se pesaron de 150 a 200 mg de muestra finamente triturada y secada previamente a $80^{\circ} \mathrm{C}$ y se disolvieron mediante la adición y evaporación $\left(125^{\circ} \mathrm{C}\right)$ repetida de ácido fluorhídrico y ácido perclórico (30:70) en bombas abiertas de teflón. En cada adición de ácido (3-4 ciclos) la solución se evaporó totalmente hasta conseguir una disolución total de la muestra. Las muestras previamente digeridas y secadas hasta evaporación se redisolvieron en $50 \mathrm{~mL}$ de solución con un $2 \%$ de $\mathrm{HNO}_{3}$ y $10 \mu \mathrm{g} \cdot \mathrm{L}^{-1} \mathrm{de}$ una solución usada como estándar interno (indio-renio). El líquido se transfirió a una botella de polipropileno para su análisis. La determinación de lantánidos se realizó mediante un ICP-MS VG-Elemental modelo PQ3. La calibración se realizó mediante soluciones elementales en agua desionizada con $2 \%$ de ácido nítrico ultrapuro. Un $65 \%$ de las muestras se midieron por duplicado y se descartaron aquellas mediciones en las que los duplicados diferían en más de un $5 \%$. Cada serie de seis muestras se midió conjuntamente con un blanco. Este proceso se ha utilizado en todas las muestras en las que sólo se reportan los elementos de las tierras raras.

Método ACTLABS: se solicitó un protocolo específico para este trabajo. Se pesaron $100 \mathrm{mg}$ de muestra y se mezclaron con tetraborato de litio ultrapuro en una proporción 1:10 y se fundieron. Las perlas fundidas fueron disueltas en ácido nítrico ultrapuro y se lavaron al final con agua destilada. Este proceso se utilizó en todas las muestras en las que se reportan otros elementos traza (indispensables para algunos diagramas de discriminación) además de los lantánidos.

En todos los casos se han realizado pruebas de precisión y exactitud por comparación con materiales estándares de referencia, obtenidos del National Institute of Standards \& Technology (NIST, USA). También se ha usado como estándar interno la roca basáltica del Cuaternario de Ciudad Universitaria (BCU3). Las mediciones de los materiales de referencia indican que la precisión de los datos para los lantánidos es mejor que $10 \%$, es decir, aproximadamente igual o menor que el tamaño de los símbolos con que se dibujan en los diagramas presentados. Los contenidos en lantánidos se normalizaron respecto a los valores de la condrita (CI) de McDonough y Sun, 1995.

\section{RESULTADOS}

A continuación se presentan los principales resultados obtenidos en las fluoritas y en los carbonatos y rocas volcánicas que actuan como la roca hospedante.

\section{Fluorita}

Los cristales de fluorita estudiados presentan, de manera general, un enriquecimiento en lantánidos ligeros respecto a los lantánidos pesados y bajas concentraciones (en general $<30$ ppm) en comparación con la roca volcánica (>100 ppm). En la Figura 2a se han proyectado nueve patrones de lantánidos de fluoritas de diferentes yacimientos de los distritos de Taxco y Zacualpan. En esta figura se observa que los tres rasgos más importantes son: a) la forma del patrón, b) la concentración total de lantánidos y c) la presencia o no de anomalías. Las concentraciones más bajas se encuentran en fluoritas asociadas a rocas carbonatadas, por ejemplo mina La Azul (muestra Az17bl, Slantánidos $=2.70 \mathrm{ppm}$ ), mina Don Baldomero (muestra

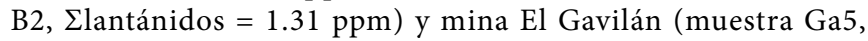
Llantánidos $=0.75 \mathrm{ppm}$ ). Las concentraciones más altas se encuentran en las fluoritas que asociadas a rocas ígneas, por ejemplo mina Los Tréboles, (muestra LT1, Llantánidos $=39.02 \mathrm{ppm}$ ), y/o rocas metamórficas, por ejemplo mina General Escobedo, (muestras Zalb, $\Sigma$ lantánidos $=33.30 \mathrm{ppm}$ y muestra Zala, Llantánidos $=24.68 \mathrm{ppm}$ ). Las muestras del distrito de Zacualpan presentan patrones más horizontales de lantánidos e incluso enriquecimiento en lantánidos pesados (p. ej., muestra Za2) respecto a los lantánidos ligeros. También la fluorita asociada a las mineralizaciones metálicas de Taxco (p. ej., muestra Tx1) está empobrecida en lantánidos ligeros respecto a la fluorita no asociada a sulfuros. A efectos únicamente comparativos se proyectan también los resultados de una muestras de fluorita bandeada del distrito minero de Las Cuevas (San Luis Potosí) en la que se observa un enriquecimiento muy marcado en lantánidos ligeros.

Se ha podido observar (Figura $2 \mathrm{~b}$ ) para la fluorita una gran variedad cromática: verde, violácea, amarilla, grisácea, etc. Las fluoritas violáceas son abundantes y por estudios previos (Pi et al., 2005a, 20015b), se conoce que son más ricas en uranio (U hasta 100 ppm) y materia orgánica. En términos generales es frecuente que la fluorita oscura (p. ej. muestras Az21, T3, Az24, Az52) presente un enriquecimiento mayor en lantánidos que la fluorita transparente o de color claro 
Tabla 1. Listado de muestras analizadas.

\begin{tabular}{|c|c|c|c|c|c|}
\hline Muestra & Localidad & Mineralogía & Textura & Generación & Color \\
\hline$A-15 v$ & La Azul & $\mathrm{Fl}(80 \%)+\mathrm{Q}(15 \%)+\mathrm{Op}+$ micas & Bandeada & $\mathrm{F} 1$ & Azul \\
\hline A-17a & La Azul & $\mathrm{Fl}$ & Masiva & F1 & Blanco \\
\hline$A-17 b$ & La Azul & $\mathrm{Fl}$ & Masiva & $\mathrm{F} 1$ & Blanco \\
\hline A-20a & La Azul & $\mathrm{Fl}(95 \%)+\mathrm{Ox}+\mathrm{Op}$ & Botroidal, cavidades de disolución & $\mathrm{F} 1$ & Azul \\
\hline$A-20 b$ & La Azul & $\mathrm{Fl}$ & Botroidal & $\mathrm{F} 1$ & Azul \\
\hline$A-20 c$ & La Azul & $\mathrm{Fl}$ & Botroidal y masiva & F1 & Azul \\
\hline A-20d & La Azul & $\mathrm{Fl}$ & Botroidal & $\mathrm{F} 1$ & Azul \\
\hline $\mathrm{Az} 2 \mathrm{v}$ & La Azul & $\mathrm{Fl}(70 \%)+\mathrm{Q}(30 \%)$ & $\begin{array}{l}\text { Ritmita, relleno de pequeñas } \\
\text { cavidades }\end{array}$ & $\mathrm{F} 1$ & Violeta \\
\hline Az3a & La Azul & $\begin{array}{l}\mathrm{Fl}(70 \%)+\mathrm{Q}(25 \%)+\mathrm{Cc}(3 \%)+ \\
\mathrm{Ox}(2 \%)\end{array}$ & $\begin{array}{l}\text { Intercrecimiento } \mathrm{Fl} \mathrm{y} \mathrm{Q} \text {, relleno } \\
\text { de cavidades }\end{array}$ & $\mathrm{F} 1$ & Violeta \\
\hline Az3b & La Azul & $\mathrm{Fl}+$ trazas de $\mathrm{Q}$ & Masiva & $\mathrm{F} 1$ & Violeta \\
\hline Az5a & La Azul & $\begin{array}{l}\mathrm{Fl}(85 \%)+\mathrm{Cb}(10 \%)+\text { trazas Op } \\
+\mathrm{Q}\end{array}$ & $\begin{array}{l}\text { Botroidal, Reemplazamiento de } \\
\mathrm{Cb} \text {, relleno de cavidades }\end{array}$ & F1 & Violeta \\
\hline Az5b & La Azul & $\mathrm{Fl}+$ trazas de $\mathrm{Q}$ & Botroidal & $\mathrm{F} 1$ & Violeta \\
\hline $\mathrm{Az} 5 \mathrm{c}$ & La Azul & $\mathrm{Fl}+$ trazas de $\mathrm{Q}$ & Botroidal & $\mathrm{F} 1$ & Violeta \\
\hline$A z 5 v$ & La Azul & $\mathrm{Fl}+$ trazas de $\mathrm{Q}$ & Botroidal & $\mathrm{F} 1$ & Violeta \\
\hline Az7 & La Azul & $\mathrm{Fl}$ & Bandeada & $\mathrm{F} 1$ & Marrón oscuro \\
\hline Az7bl & La Azul & $\mathrm{Fl}$ & Bandeada & $\mathrm{F} 1$ & Blanco \\
\hline Az7n & La Azul & $\mathrm{Fl}$ & Bandeada & $\mathrm{F} 1$ & Marrón \\
\hline Az17bl & La Azul & $\mathrm{Fl}$ & Masiva (cristales milimétricos) & $\mathrm{F} 2$ & Blanco \\
\hline Az17c & La Azul & $\mathrm{Fl}$ & Masiva (cristales milimétricos) & F2 & Marrón claro \\
\hline $\mathrm{Az} 20 \mathrm{v}$ & La Azul & $\mathrm{Fl}$ & Costra & $\mathrm{F} 1$ & Azul \\
\hline Az21 & La Azul & FL (95\%) trazas de Q y Cc & $\begin{array}{l}\text { Bandeada, pequeñas zonas con } \\
\text { disolución }\end{array}$ & $\mathrm{F} 1$ & Blanco/negro \\
\hline Az21a & La Azul & $\mathrm{Fl}$ & Bandeada & $\mathrm{F} 1$ & Marrón \\
\hline Az21b & La Azul & $\mathrm{Fl}$ & Bandeada & & Marrón \\
\hline Az24n & La Azul & $\mathrm{Fl}(95 \%)$ trazas de Ox $+\mathrm{Op}$ & Bandeada, capas concéntricas & $\mathrm{F} 1$ & Marrón oscuro \\
\hline Az25 & La Azul & $\mathrm{Fl}$ & Costra, texturas de disolución & $\mathrm{F} 2$ & Blanco, azul muy claro \\
\hline Az45bl & La Azul & $\mathrm{Fl}$ & Masiva & $?$ & Blanco y violeta \\
\hline Az48 & La Azul & $\mathrm{Fl}+$ trazas de $\mathrm{Cc}$ & Relleno de cavidades & F2 & Variable \\
\hline Az52a & La Azul & $\mathrm{Fl}$ & Microcristalina y masiva & $\mathrm{F} 1$ & Variable \\
\hline Az52b & La Azul & $\mathrm{Fl}$ & Microcristalina y masiva & $\mathrm{F} 1$ & Variable \\
\hline Az53a & La Azul & $\mathrm{Fl}+\mathrm{Q}$ & Brecha & $?$ & Variable \\
\hline Az53b & La Azul & $\begin{array}{l}\mathrm{Fl}(80 \%)+\mathrm{Q}+\mathrm{Op}+\mathrm{Ox}+\text { restos } \\
\text { de mica }\end{array}$ & Brecha & F2 & Variable \\
\hline Az54bl & La Azul & $\mathrm{Fl}+\mathrm{Q}$ & Brecha & $?$ & Variable \\
\hline Az92m & La Azul & $\mathrm{Fl}(95 \%)+\mathrm{Q}+\mathrm{Cc}$ & Bandeada & $\mathrm{F} 1$ & Marrón \\
\hline $\mathrm{Az} 92 \mathrm{v}$ & La Azul & $\mathrm{Fl}(90 \%)+\mathrm{Q}+\mathrm{Cc}+\mathrm{Ox}$ & Bandeada & $\mathrm{F} 1$ & Violeta \\
\hline Az100 & La Azul & $\mathrm{Fl}$ & Masiva & F1 & Transparente \\
\hline Az101 & La Azul & $\mathrm{Fl}$ & Relleno de cavidades & $\mathrm{F} 1$ y F2 & Transparente \\
\hline $\mathrm{N} 1 \mathrm{Bv}$ & La Azul & $\mathrm{Fl}$ & Capa con cristales milimétricos & & Violeta oscuro \\
\hline $\mathrm{N} 2 \mathrm{v}$ & La Azul & $\mathrm{Fl}+\mathrm{Q}$ & Masiva & & Violeta oscuro \\
\hline $\mathrm{N} 3 \mathrm{v}$ & La Azul & $\mathrm{Fl}+\mathrm{Q}+\mathrm{Ba}+\mathrm{Op}$ & Masiva & & Violeta oscuro \\
\hline N4B1v & La Azul & $\mathrm{Fl}+\mathrm{Ox}$ & Cristales milimétricos & & Violeta oscuro \\
\hline N4B2 & La Azul & $\mathrm{Fl}+\mathrm{Ox}$ & Cristales milimétricos & & Violeta oscuro \\
\hline N5A & La Azul & $\mathrm{Fl}$ & Nódulo concéntrico & & Violeta oscuro \\
\hline N5B & La Azul & $\mathrm{Fl}+(\mathrm{Cb})$ & Nódulo concéntrico & & Violeta oscuro \\
\hline $\mathrm{Nv}$ & La Azul & $\mathrm{Fl}$ & Nódulo & & Violeta oscuro \\
\hline T1a & La Azul & $\mathrm{Fl}+\mathrm{Q}+\mathrm{Ba}+\mathrm{Op}+\mathrm{Ox}$ & $\begin{array}{l}\text { Reemplazamiento de carbonato, } \\
\text { intercrecimiento } \mathrm{Fl} \mathrm{y} \mathrm{Q}\end{array}$ & $\mathrm{F} 1$ & Violeta \\
\hline $\mathrm{T} 1 \mathrm{~b}$ & La Azul & $\mathrm{Fl}+\mathrm{Q}+\mathrm{Ba}+\mathrm{Op}+\mathrm{Ox}$ & $\begin{array}{l}\text { Reemplazamiento de carbonato, } \\
\text { intercrecimiento } \mathrm{Fl} \mathrm{y} \mathrm{Q}\end{array}$ & $\mathrm{F} 1$ & Violeta \\
\hline T1c & La Azul & $\mathrm{Fl}+\mathrm{Q}+\mathrm{Ba}+\mathrm{Op}+\mathrm{Ox}$ & $\begin{array}{l}\text { Reemplazamiento de carbonato, } \\
\text { intercrecimiento Fl y Q }\end{array}$ & $\mathrm{F} 1$ & Violeta \\
\hline $\mathrm{T} 1 \mathrm{v}$ & La Azul & $\mathrm{Fl}+\mathrm{Q}+\mathrm{Ba}+\mathrm{Op}+\mathrm{Ox}$ & $\begin{array}{l}\text { Reemplazamiento de carbonato, } \\
\text { intercrecimiento Fl y Q }\end{array}$ & $\mathrm{F} 1$ & Violeta \\
\hline
\end{tabular}

Notas: $\mathrm{Fl}=$ fluorita, $\mathrm{Q}=$ cuarzo, $\mathrm{Cc}=$ calcita, $\mathrm{Op}=$ minerales opacos, $\mathrm{Ox}=$ óxidos de hierro, $\mathrm{Cb}=$ carbonato, $\mathrm{Ba}=$ barita. 
Tabla 1 (continuación). Listado de muestras analizadas.

\begin{tabular}{|c|c|c|c|c|c|}
\hline Muestra & Localidad & Mineralogía & Textura & Generación & Color \\
\hline T3a & La Azul & $\mathrm{Fl}+\mathrm{Q}+\mathrm{Cc}$ & Brecha & $\mathrm{F} 1$ & Violeta \\
\hline $\mathrm{T} 3 \mathrm{~b}$ & La Azul & $\mathrm{Fl}+\mathrm{Q}+\mathrm{Cc}$ & Brecha & $\mathrm{F} 1$ & Violeta \\
\hline $\mathrm{T} 3 \mathrm{v}$ & La Azul & $\mathrm{Fl}+\mathrm{Q}+\mathrm{Cc}$ & Brecha & $\mathrm{F} 1$ & Violeta \\
\hline T7 & La Azul & $\mathrm{Cc}$ & Bandeada & $\mathrm{F} 1$ & Blanco y gris \\
\hline Cuevgr & Las Cuevas & $\mathrm{Fl}$ & Bandeada & & Amarillo \\
\hline Cuevm & Las Cuevas & & Bandeada & & Marrón \\
\hline B-2 & Don Baldomero & $\mathrm{Fl}+\mathrm{Q}$ & Relleno de cavidades & $\mathrm{F} 2$ & Azul-verdoso \\
\hline F1Tx & Taxco & Fl (asociada a galena) & Cristal de $1 \mathrm{~cm}$ & & Transparente \\
\hline $\mathrm{Ga}-5$ & El Gavilán & $\mathrm{Fl}+$ Caolín & Masiva & & Rojo \\
\hline LT-1 & La Azul & $\mathrm{Q}+\mathrm{Fl}+$ Caolín $+\mathrm{Cc}+\mathrm{Ox}$ & Brechoide & & Verdoso \\
\hline Zala & Zacualpan & $\mathrm{Fl}$ & Cristalina & Za1 & Blanco Translucido \\
\hline Zalb & Zacualpan & $\mathrm{Fl}$ & Cristalina & Za1 & Blanco Translucido \\
\hline Zalbl & Zacualpan & $\mathrm{Fl}$ & Cristalina & Za1 & Blanco \\
\hline $\mathrm{Za} 2$ & Zacualpan & $\mathrm{Fl}$ & Cristalina & Za1 & Blanco \\
\hline $\mathrm{Za} 2 \mathrm{Bl}$ & Zacualpan & $\mathrm{Fl}$ & Cristalina & Za1 & Blanco \\
\hline Az12 & La Azul & Roca volcánica & Porfídica & & Rojo \\
\hline $\mathrm{Ga}-4 \mathrm{a}$ & El Gavilán & Roca volcánica & Roca volcánica alterada & & Rojo \\
\hline $\mathrm{Ga}-4 \mathrm{c}$ & El Gavilán & Roca volcánica & Roca volcánica alterada & & Rojo \\
\hline Tx7 & La Azul & Roca volcánica & Porfídica & & Rojo \\
\hline Tx8 & La Azul & Roca volcánica & Porfídica & & Rojo \\
\hline Tx10 & La Azul & Roca volcánica & Porfídica & & Rojo \\
\hline Az83a & La Azul & Arcillas + Q & Sin textura & & Rojo \\
\hline Az83b & La Azul & Arcillas + Q & Sin textura & & Rojo \\
\hline Az83c & La Azul & Arcillas + Q & Sin textura & & Rojo \\
\hline $\begin{array}{l}\text { A14 } \\
\text { barita }\end{array}$ & La Azul & $\mathrm{Ba}$ & Masiva & & Blanco \\
\hline $\begin{array}{l}\mathrm{Az}- \\
49 \mathrm{barita}\end{array}$ & La Azul & $\mathrm{Ba}$ & Masiva & & Blanco \\
\hline $\mathrm{A} 54 \mathrm{cb}$ & La Azul & $\mathrm{Cc}$ & Masiva & & Gris \\
\hline $\mathrm{Az}-22 \mathrm{cb}$ & La Azul & $\mathrm{Cc}$ & Bandeada & & Marrón \\
\hline $\mathrm{Ga}-4 \mathrm{~b}$ & El Gavilán & $\mathrm{Cc}$ & Irregular & & Variable \\
\hline T52a & La Azul & $\mathrm{Cc}$ & Masiva & & Gris \\
\hline T52b & La Azul & $\mathrm{Cc}$ & Masiva & & Gris \\
\hline T6 & La Azul & $\mathrm{Cc}$ & Masiva & & Gris \\
\hline T7 & La Azul & $\mathrm{Cc}$ & Masiva & & Gris \\
\hline
\end{tabular}

Notas: $\mathrm{Fl}=$ fluorita, $\mathrm{Q}=$ cuarzo, $\mathrm{Cc}=$ calcita, $\mathrm{Op}=$ minerales opacos, $\mathrm{Ox}=$ óxidos de hierro, $\mathrm{Cb}=\mathrm{carbonato}, \mathrm{Ba}=\mathrm{barita}$.

(p. ej., muestras Az17 y Az7bl). Esto puede observarse también en las fluoritas de otros distritos mineros como el de Las Cuevas en el que la fluorita amarilla (CuevGr) es mucho más pobre en lantánidos (cerca del límite de detección del ICP-MS) que la fluorita de color marrón (CuevMa). Sin embargo, no se puede establecer para todas las muestras una correlación univoca entre el color y la concentración de lantánidos ya que algunas muestras de color claro (Az45) más tardías, presentan una concentración mayor que las muestras tempranas más oscuras.

\section{Carbonato}

En general los carbonatos sedimentarios presentan bajos contenidos en lantánidos y patrones bastante horizontales. Los carbonatos hidrotermales están asociados a las vetas de fluorita (p. ej. Los Tréboles, Ilantánidos $\mathrm{Cb}_{\text {hidrotermal }}=27 \mathrm{ppm}$ ) y siempre tienen concentraciones más elevadas de lantánidos que los carbonatos de origen sedimentario (p. ej. La Azul, Llantánidos $\mathrm{Cb}_{\text {sedimentario }}=0.3-5 \mathrm{ppm}$ ), y pueden presentar anomalías de Eu (Figura 3).

\section{Barita}

Los minerales de barita analizados presentan patrones con un mínimo enriquecimiento en $\Sigma$ lantánidos $(<4 \mathrm{ppm})$ y un anomalía positiva de Eu muy importante (Figura 3 ).
Riolitas de la secuencia volcánica encajonante (Distrito Taxco)

Los patrones de lantanidos de todas las rocas volcanicas asociadas a las mineralizaciones de fluorita son muy similares (Figura 3), están más enriquecidos en $\Sigma$ lantánidos (136 a 160 ppm) que los minerales estudiados (fluorita, barita, calcita) y presentan una anomalía negativa de Eu muy característica de los procesos de cristalización fraccionada de plagioclasa. Es interesante remarcar que las rocas volcánicas mineralizadas (Az12, Ga4a y Ga4c) se caracterizan por presentar un empobrecimiento general en lantánidos respecto a la roca original no alterada.

Un ejemplo interesante en que se ve la influencia del encajonante es el de la mineralización de El Gavilán (Figura 4) asociada a rocas volcánicas del Cenozoico. La fluorita presenta una anomalía positiva de Eu mientras que la calcita hidrotermal y la roca volcánica presentan anomalía negativa en dicho elemento y un mayor enriquecimiento en lantánidos. Las riolitas de la roca volcánica mineralizada presentan un patrón intermedio entre el de la roca volcánica no alterada y el de la fluorita.

\section{Alteración argílica}

El contenido en lantánidos de las arcillas (identificadas por DRX como de tipo caolínítico) asociadas a las alteraciones argílicas de los 

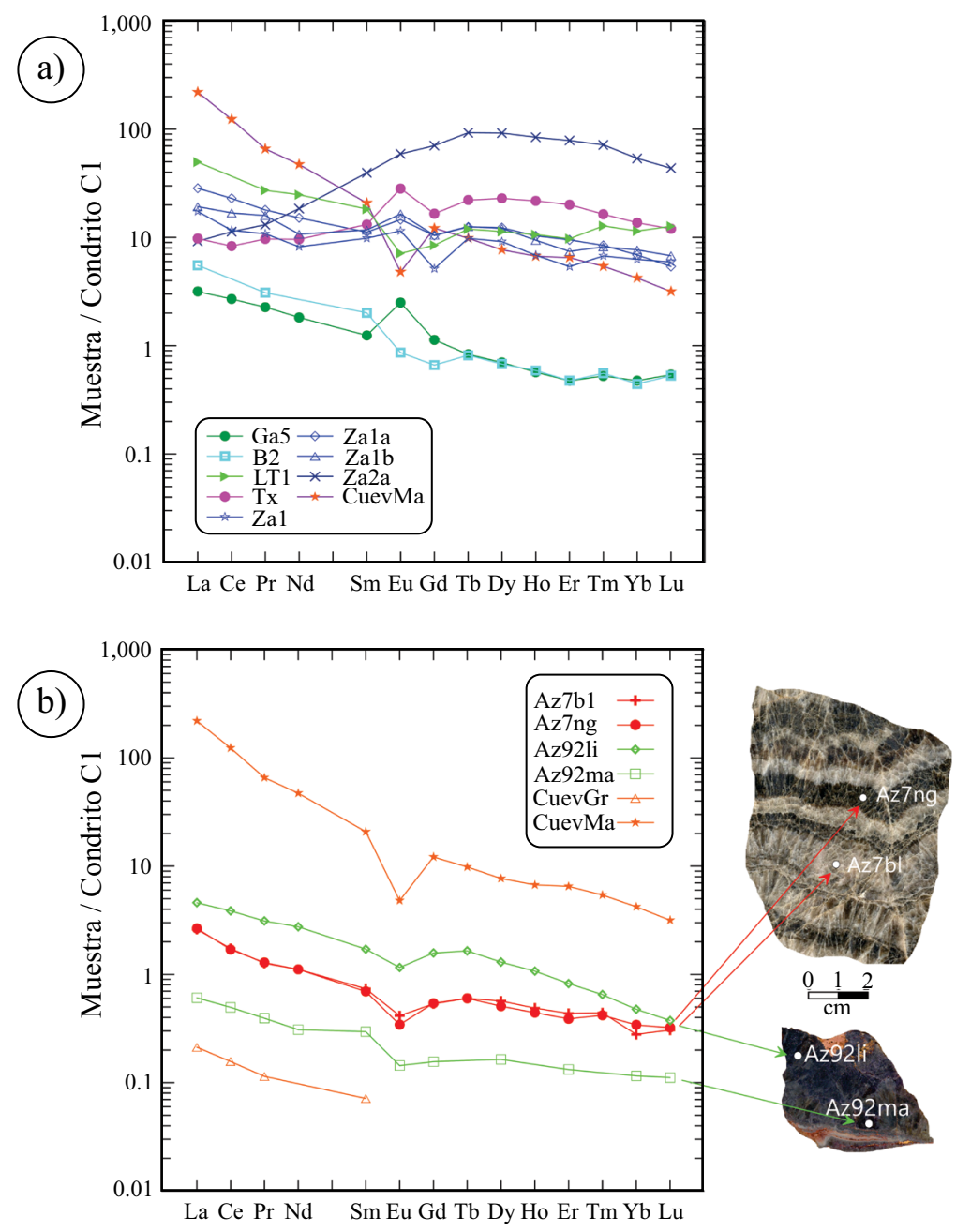

Figura 2. a) Patrones de lantánidos de fluoritas de diferentes yacimientos y distritos mineros: Ga5: El Gavilán, yacimiento del distrito de Taxco asociado al reemplazo de carbonato. B2: Don Baldomero, yacimiento del distrito de Taxco asociado al reemplazo de carbonato. LT1: Los Tréboles, yacimiento del distrito de Taxco asociado a rocas volcánicas. Tx: fluorita del yacimiento de $\mathrm{Pb}-\mathrm{Ag}-\mathrm{Zn}$ del distrito minero de Taxco. Za1, Za1a, Zalb y Za2a: vetas hidrotermales de fluorita encajadas en rocas metamórficas del distrito minero de Zacualpan. CuevMa: variedad de color marrón de la fluorita del distrito minero de Las Cuevas. b) Patrones de lantánidos de bandas de diferente color de dos muestras de fluorita del distrito minero de Taxco (Az7 y Az92) y una del distrito minero de Las Cuevas (Cuev). Az7bl: fluorita color blanco, Az7ng: fluorita color negro, Az92li: fluorita color violáceo, Az92ma: fluorita color marrón, CuevGr: fluorita amarilla, CuevMa: fluorita color marrón.

yacimientos es variable ( 55 a $90 \mathrm{ppm}$ ). En la Figura 3 se observa que estas arcillas presentan un patrón paralelo al de la roca volcánica pero con concentraciones menores de lantánidos.

\section{Fluorita asociada a sulfuro}

Algunos parámetros importantes para diferenciar los depósitos de fluorita asociados a sulfuros son los que nos relacionan el contenido de lantánidos ligeros respecto al contenido de lantánidos pesados.

Las relaciones $\mathrm{La} / \mathrm{Yb}, \% \mathrm{LaCeNd}$ (porcentaje de estos tres elementos respecto a la suma de todos los lantánidos) y LREE/HREE son siempre mayores para las fluoritas de yacimientos sin sulfuros (La/Yb de 1.9 a 17.5, \%LaCeNd de 57 a 83 y LREE /HREE entre 2.5 y 15) que para las fluoritas que se encuentran asociadas a minerales metálicos de los distritos de Taxco $(\mathrm{La} / \mathrm{Yb}=0.7, \% \mathrm{LaCeNd} \sim 35$ y LREE/HREE $<1)$ y Zacualpan $(\mathrm{La} / \mathrm{Yb}<5, \% \mathrm{LaCeNd}<60$ y LREE/HREE $<3)$.

Para las fluoritas de las mineralizaciones metálicas de Taxco y de Zacualpan la relación $\mathrm{Eu} / \mathrm{Eu}^{*}$ definida como $\left[\mathrm{Eu} /\left(\mathrm{Sm}^{*} \mathrm{Gd}\right)^{1 / 2}\right]$ es más alta que para la fluorita no asociada a sulfuros, con excepción de las escasas fluoritas con anomalía positiva de Eu.

\section{Diagrama $S m-L a$}

Para todas las muestras de fluorita y de carbonatos sedimentarios se observa una correlación positiva muy acusada entre las concentraciones de estos lantánidos (Figura 5a). En general los valores más bajos son los del carbonato.
Las fluoritas asociadas a sulfuros presentan contenidos en La y Sm intermedios entre los de la fluorita de yacimientos monominerales y los de las rocas de caja volcánicas y una correlación negativa entre el contenido en ambos.

Diagramas $\mathrm{Eu} / \mathrm{Eu^{* }}-\mathrm{La}$

Este diagrama (Figura 5b) permite discriminar la fluorita violácea rica en uranio y asociada a barita (anomalía negativa de Eu muy acusada) y observar que los valores de la relación $\mathrm{Eu} / \mathrm{Eu}^{*}$ son en general superiores (mayores a 1) en la fluorita asociada a sulfuros (Taxco y Zacualpan). Las fluoritas relacionadas con las rocas volcánicas (p. ej., Mina Los Tréboles) quedan dentro del campo de las rocas volcánicas y los yacimientos relacionados con el reemplazo de carbonato (Minas Don Baldomero y El Gavilán) se proyectan cerca del campo de los carbonatos.

\section{DISCUSIÓN E INTERPRETACIÓN}

\section{Formación de complejos}

La formación de complejos fluorados de lantánidos en las soluciones acuosas es uno de los factores que más puede influir en la distribución de lantánidos en fluoritas. Experimentalmente se ha demostrado que la estabilidad química de los complejos fluorados de lantánidos crece del La al Lu (p. ej., Möller y Morteani, 1983; Bau 


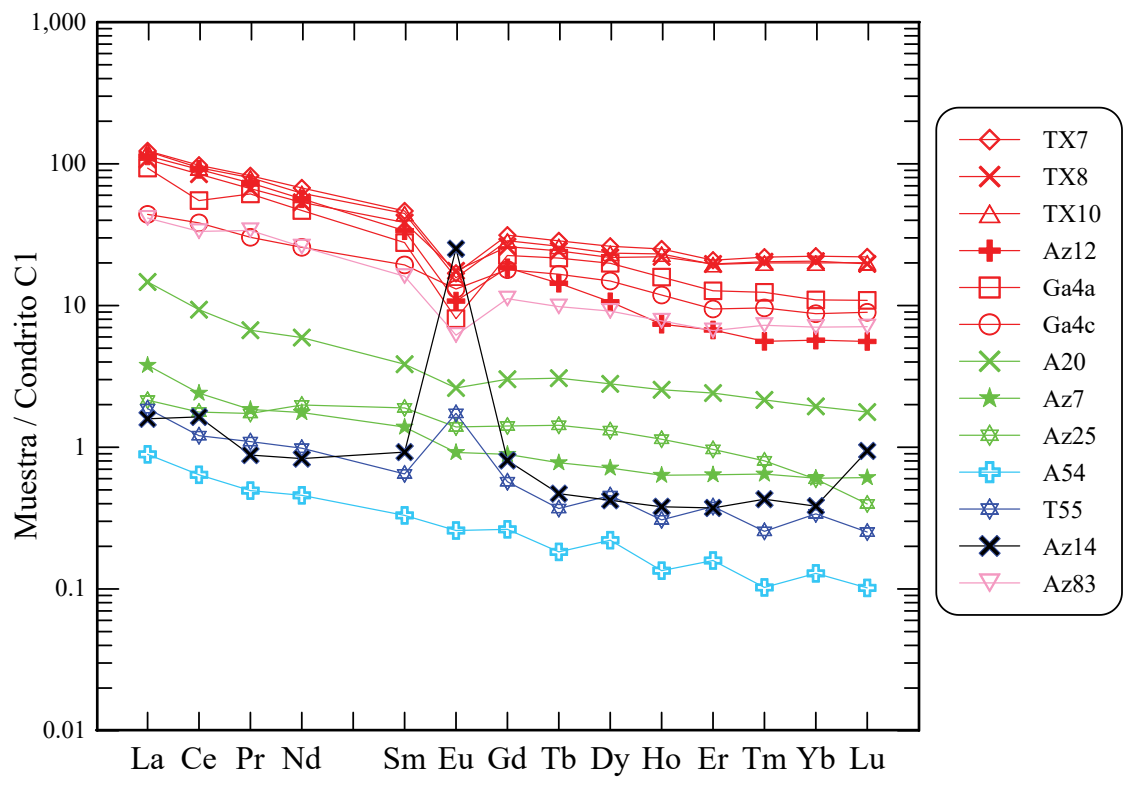

Figura 3. Patrones de lantánidos de rocas y minerales del distrito minero de Taxco: rocas volcánicas sin contacto con las mineralizaciones (Tx7, Tx8, Tx10), rocas volcánicas encajonantes de las mineralizaciones de fluorita La Azul y El Gavilán (Az12, Ga4a), roca volcánica mineralizada de la mina el Gavilán (Ga4c), diferentes generaciones de fluorita de la mina La Azul (A20, Az7, Az25), carbonato sedimentario (A54) de la Formación Morelos, carbonato hidrotermal (T55) asociado a las mineralizaciones de fluorita de la mina La Azul, barita de la mina La Azul (Az14) y alteración argílica (Az83) asociada a las mineralizaciones.

et al., 2003). También los datos termodinámicos sobre la estabilidad de complejos de lantánidos en soluciones acuosas. (p. ej., Haas et al., 1993) sugieren que la especiación de los lantánidos en soluciones que precipitan fluorita está controlada por la presencia de estos complejos fluorados. En condiciones ácidas el patrón de lantánidos del fluido se ve controlado por procesos de absorción y obtenemos patrones con relaciones $\mathrm{La} / \mathrm{Lu}$ mayores a 1 y el Eu se encuentra básicamente como $\mathrm{Eu}^{2+}$. En medios neutros a básicos el patrón de lantánidos está determinado por los procesos de formación de complejos con carbonatos, flúor e hidróxidos y en general se tienen patrones con relaciones $\mathrm{La} / \mathrm{Lu}$ inferiores a 1. En este caso crece el campo de estabilidad del $\mathrm{Eu}^{3+}$ y sólo se forma $\mathrm{Eu}^{2+}$ en condiciones muy reductoras.

Para los yacimientos estudiados (especialmente en el distrito de Taxco) se observa un predominio de fluorita con relaciones $\mathrm{La} / \mathrm{Lu}$ mayores a 1 lo que se relaciona con el predominio en las etapas iniciales de la mineralización de fluidos de naturaleza ácida.

\section{Parámetros geoquímicos}

La comparación de los patrones de lantánidos no es simple por lo que se introducen diferentes relaciones geoquímicas entre lantánidos (Tabla A1, disponible como suplemento electrónico), que sirven para cuantificar el grado de fraccionamiento y que permiten diferenciar si este fraccionamiento está asociado a la evolución de la mineralización o se debe a procesos de remobilización.

En todas las fluoritas analizadas no asociadas a sulfuros obtenemos valores variables pero siempre mayores que 1 (1.9 a 17.5) de la relación $\mathrm{La} / \mathrm{Yb}$. En la roca volcánica alterada se observan valores más altos de esta relación $(\sim 20)$ y en los carbonatos dicha relación presenta un rango más amplio ( 4 a 42). Únicamente para las fluoritas asociadas a sulfuro de ambos distritos se obtienen valores más pequeños de esta relación ( 0.7 en Taxco y $<5$ en Zacualpan). La relación $\mathrm{La} / \mathrm{Lu}$ varia de 2 a 19 para las fluoritas de los depósitos sin sulfuros de Taxco, es muy similar a la relación $\mathrm{La} / \mathrm{Lu}$ de los carbonatos hidrotermales (4 a 19) y es más variable que la relación $\mathrm{La} / \mathrm{Lu}$ de las rocas volcánicas (4 a 10).
Una relación $\mathrm{La} / \mathrm{Lu}$ mayor a 1 se considera típica de soluciones iónicas ya que en estas el fraccionamiento es poco importante pues las diferencias de radio iónico entre lantánidos ligeros y pesados son pequeñas. Esta relación $\mathrm{La} / \mathrm{Lu}$ disminuye claramente en las fluoritas asociadas a sulfuros ( 0.8 en Taxco y $<3$ en Zacualpan). Esto puede indicarnos la presencia en el fluido de cantidades importantes de $\mathrm{CO}_{3}{ }^{2-}, \mathrm{F}^{-}$y $\mathrm{OH}^{-}$ que forman compuestos más estables con los lantánidos pesados que con los ligeros, produciendo un fraccionamiento importante durante la migración del fluido y un enriquecimiento en la relación $\mathrm{Y} / \mathrm{Ho}$ (Krupenin et al., 1999).

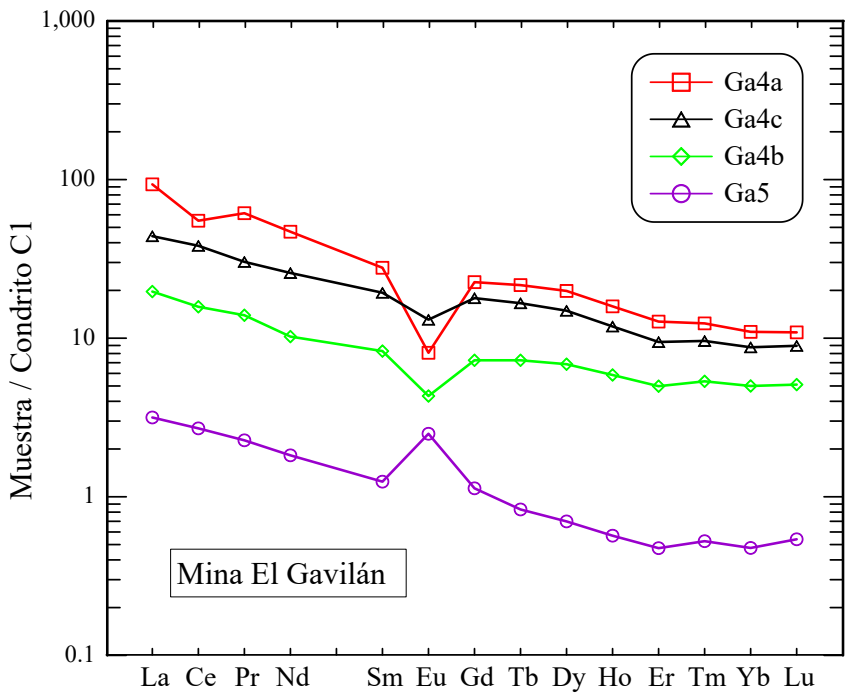

Figura 4. Patrones de lantánidos de la mina El Gavilán del distrito minero de Taxco: roca volcánica (Ga4a), roca volcánica mineralizada (Ga4c), calcita hidrotermal (Ga4b) y fluorita (Ga5). 


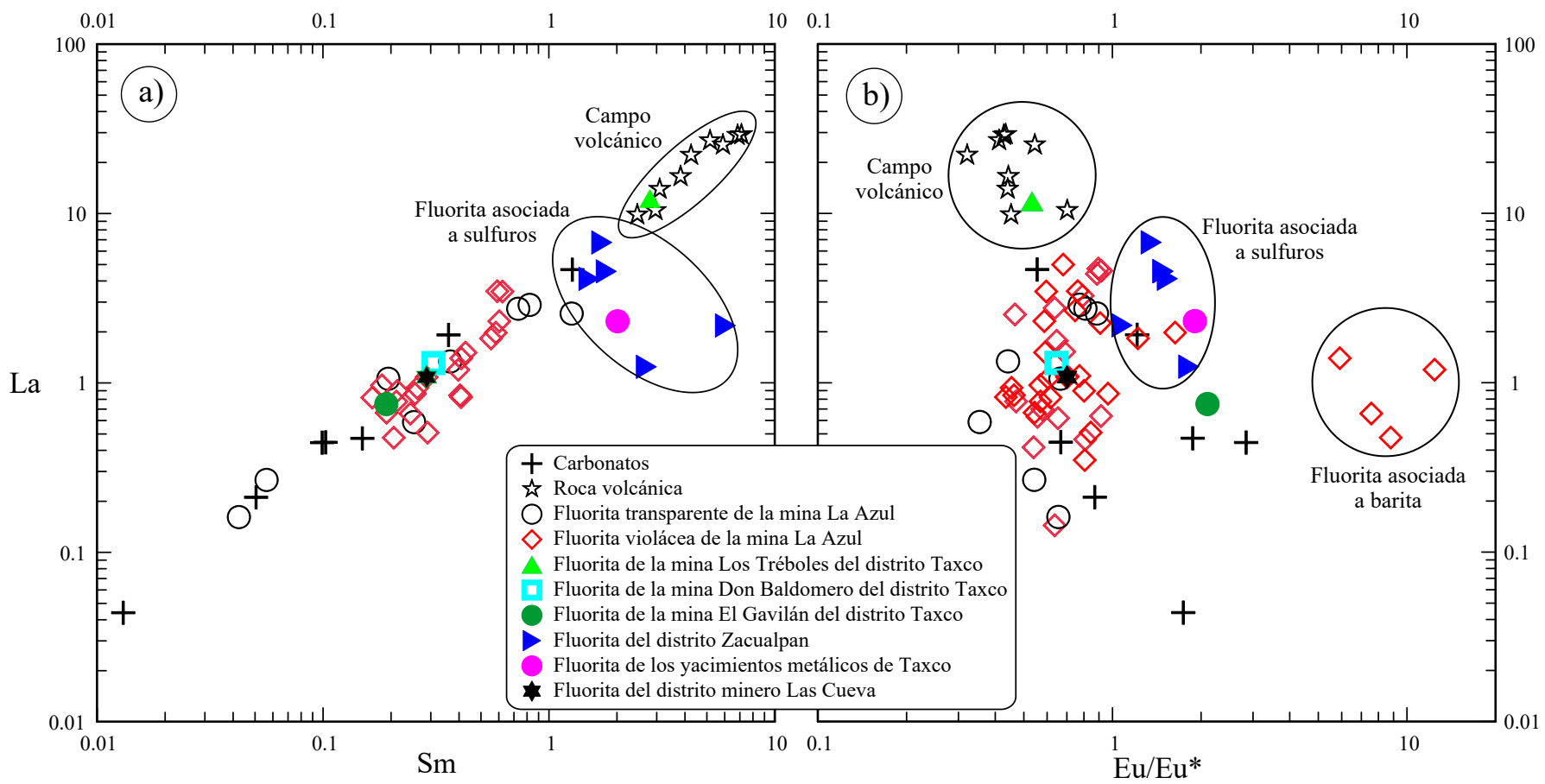

Figura 5. a) Diagrama La - Sm, b) Diagrama La - Eu/Eu*

En algunos casos existe una variación importante de la relación La/Ho (Tabla A1 del suplemento electrónico) que sugiere posibles procesos postdeposicionales (Bau y Dulski, 1995) como los de disolución-recristalización que se han confirmado en el estudio textural y mineralógico detallado de las mineralizaciones.

Möller et al., (1976) basándose en las relaciones Tb/Ca y Tb/La definen tres tipos genéticos de fluoritas: pegmatíticas (valores más altos de estas relaciones), hidrotermales (valores intermedios) y sedimentarias (valores más bajos). Según estos autores la relación $\mathrm{Tb} / \mathrm{La}$ puede considerarse un índice de fraccionamiento (máximo en fluorita de cuerpos pegmatíticos y mínimo en fluorita sedimentaria) ya que el La, que es el más abundante de los lantánidos trivalentes, es extraído pronto de la solución con lo que la relación $\mathrm{Tb} / \mathrm{La}$ crece en las fluoritas tardías. La relación $\mathrm{Tb} / \mathrm{Ca}$ puede considerarse un parámetro ambiental que sirve para identificar si se han producido procesos de reequilibrio con un reservorio de tipo sedimentario. En todos los casos se obtuvieron relaciones $\mathrm{Tb} / \mathrm{La}$ y $\mathrm{Tb} / \mathrm{Ca}$ bastante bajas (Figura 6). Con el término fluoritas sedimentarias se hace referencia a fluoritas relacionadas genéticamente con el reemplazo de carbonatos pero asociadas a un fluido hidrotermal relacionado con actividad magmática. En las fluoritas no asociadas a sulfuros del distrito minero de Taxco la relación $\mathrm{Tb} / \mathrm{La}$ va de 0.1 a 1.2 con predominio de valores bajos ( 0.2 a 0.5$)$. Sólo en el caso de las mineralizaciones de fluorita asociada a sulfuros la relación $\mathrm{Tb} / \mathrm{La}$ es más alta (Taxco Tb/La > 2 y Zacualpan Tb/La hasta 10 ).

Según Bau (1996), las relaciones Y/Ho y Zr/Hf pueden ser usadas para determinar cuando los minerales deben relacionarse con fundidos silicatados o fluidos acuosos. En medios acuosos dichos elementos con carga y radio muy similares forman complejos y su distribución vendrá controlada en parte por la configuración electrónica y tipo de enlace químico. La fluorita derivada de sistemas magmáticos mostrará una relación Y/Ho aproximadamente condrítica ( 27.5, Barrat et al., 2012). Las muestras de fluorita hidrotermal se caracterizan por una asociación muy estrecha del Y con los lantánidos y una relación Y/ Ho (>200) no condrítica. Esto se debe a que el Y y el Ho se fraccionan mutuamente en fluidos ricos en flúor de temperatura media-alta
(Walker y Chopin, 1967). A medida que se incrementa la distancia de migración se observa una anomalía positiva de Y (Bau y Dulski, 1995). Para algunas muestras (p. ej. Az7 y Az21), se obtienen relaciones Y/Ho y Zr/Hf (Figura 7) aproximadamente condríticas (sin anomalía de Y) lo que indica que dicha fluorita se puede correlacionar genéticamente con fluidos que derivan de fundidos silicatados (Bau y Dulski, 1995) de origen magmático. Otras fluoritas presentan valores más altos de la relación $\mathrm{Y} / \mathrm{Ho}$ demostrando que existe un cierto grado de migración del fluido mineralizante respecto a la fuente magmática y una tendencia bien marcada hacia el campo que se considera característico de la fluorita hidrotermal. Las fluoritas de Taxco se sitúan en general más cerca del campo magmático y las del distrito Zacualpan se aproximan al campo de fluoritas hidrotermales.

\section{Anomalías de europio}

Con excepción de algunas muestras (p. ej., T1, Az-3 y Az-5) la relación $\mathrm{Eu} / \mathrm{Eu}^{*}$ de la mayoría de las fluoritas asociadas a depósitos de fluorita sin sulfuros, da en un rango muy estrecho (0.4-0.9). Para las fluoritas de las mineralizaciones metálicas de Taxco (1.9) y de Zacualpan (1 a 1.4) este valor es mayor a 1 (anomalía positiva de Eu). Los datos termodinámicos en sistemas acuosos (Sverjensky, 1984) demuestran que a presión elevada y a temperatura mayor de $250{ }^{\circ} \mathrm{C}$ predomina el $\mathrm{Eu}^{2+}$ mientras que en ambientes superficiales y de baja temperatura $\left(25^{\circ} \mathrm{C}\right.$ y 1 bar $)$ dicho elemento se encuentra básicamente en estado trivalente y la reducción del $\mathrm{Eu}^{3+}$ sólo sería factible en condiciones de muy baja fugacidad de oxígeno y con un $\mathrm{pH}$ próximo a 7 . $\mathrm{Si}$ el Eu está como $\mathrm{Eu}^{3+}$ se concentra en el fluido (es altamente soluble) en mayor proporción que el La, $\mathrm{Nd}$, Ga y Lu y se produce una anomalía negativa de $\mathrm{Eu}$ en los minerales. Si está como $\mathrm{Eu}^{2+}$ (es poco soluble), entra en el mineral (anomalía positiva) y determinará la formación de anomalías negativas de Eu en la solución. El potencial redox $\mathrm{Eu}^{3+} / \mathrm{Eu}^{2+}$ depende fuertemente de la temperatura y en menor grado del $\mathrm{pH}$ y de la presión. De esta forma, el simple enfriamiento de las soluciones de origen profundo puede determinar un cambio del estado de oxidación del Eu y anomalías de diferente signo en la fluorita. 


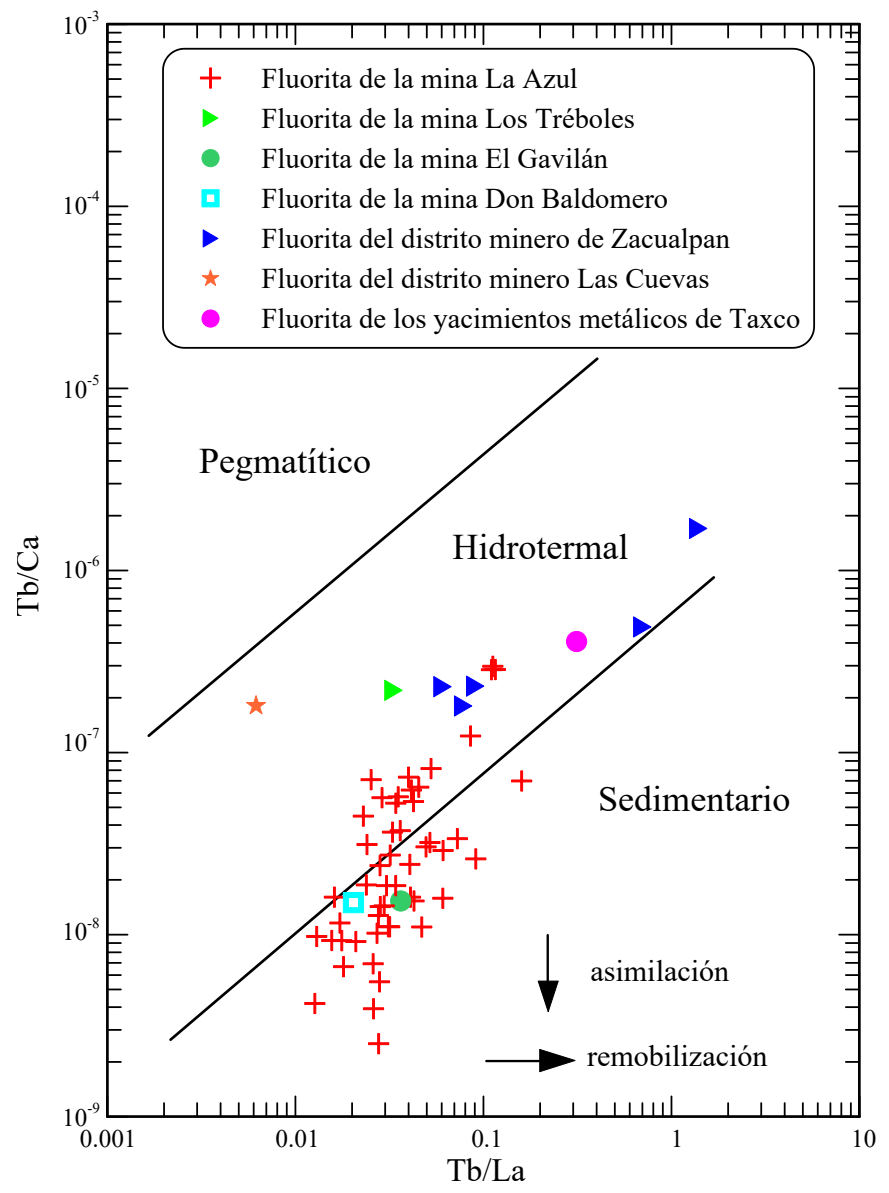

Figura 6. Diagrama Tb/Ca - Tb/La (Möller et al., 1976).

Evolución de los fluidos mineralizantes en la mina La Azul

Se propone un modelo de la evolución del fluido mineralizante en el caso de las fluoritas de la mina La Azul por ser este depósito tanto el de mayor tamaño como el único en que se han podido discriminar diferentes episodios de mineralización (Figura 8 a y 8 b):

1. Presencia de un fluido ácido, con temperatura superior a los $250{ }^{\circ} \mathrm{C}$ (asociado a la alteración argilítica de las rocas de caja) y reductor, rico en $\mathrm{F}$ y pobre en Ca que precipita fluorita (F1a) violácea rica en materia orgánica, $\mathrm{H}_{2} \mathrm{~S}$, óxidos de uranio y anomalía positiva de $\mathrm{Eu}$, por interacción y reemplazamiento del carbonato de la formación Morelos. Las texturas de la fluorita Fla son primarias (masiva, botroidal o rítmica).

2. En una segunda etapa, casi simultánea a la anterior, este fluido precipita barita con un patrón típico de las baritas continentales y anomalía positiva de Eu muy acusada.

3. Desciende la temperatura del fluido y por interacción con los carbonatos el fluido se neutraliza. Crece el campo de estabilidad del $\mathrm{Eu}^{3+}$ sin que haya cambios muy grandes de fugacidad de oxígeno. Precipitan la fluoritas F1b y F1c con anomalía negativa de Eu.

4. Por entrada de nuevos fluidos en el sistema precipitan fluoritas más tardías, con texturas secundarias que se denominan fluoritas F2 y en general son de color más claro, no están asociadas al reemplazo del carbonato, están más enriquecidas en lantánidos y muestran texturas típicamente secundarias. F2a es una fluorita enriquecida en lantánidos que no presenta anomalía significativa de Eu. F2b es una fluorita con contenidos intermedios de lantánidos que forma principalmente nódulos y capas externas de fluorita tardía y secundaria.

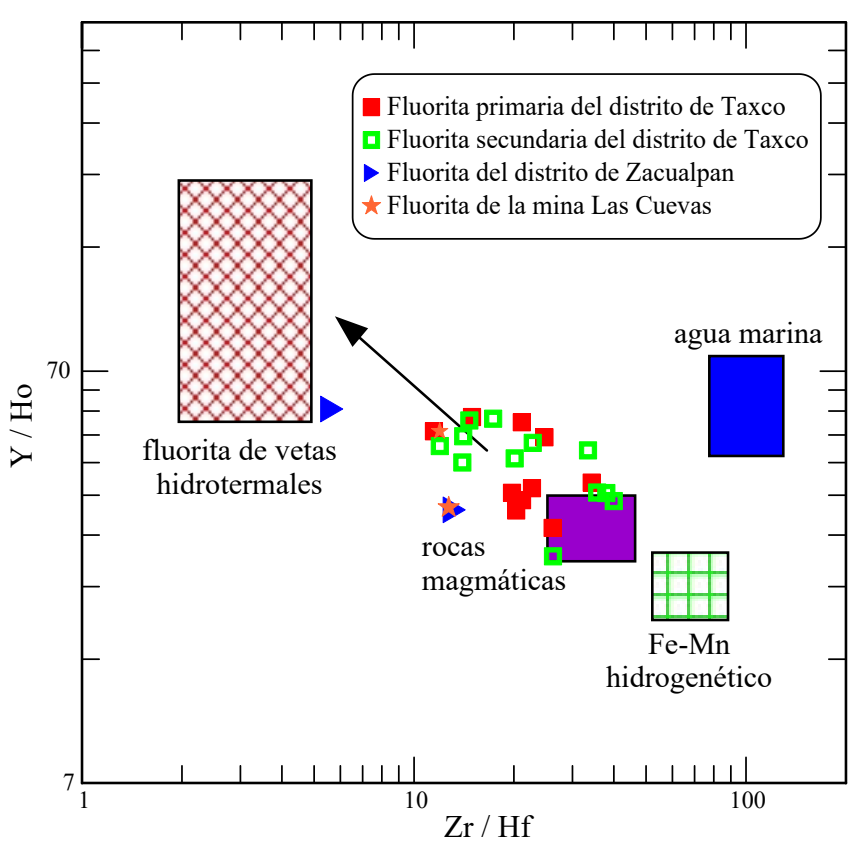

Figura 7. Gráfico Y/Ho - Zr/Hf (Möller et al., 1976).

\section{CONCLUSIONES}

Los cristales de fluorita estudiados tienen, en general, un enriquecimiento en lantánidos ligeros respecto a los lantánidos pesados y presentan bajas concentraciones en lantánidos en comparación con la roca volcánica o metamórfica. Las concentraciones más bajas en lantánidos se encuentran en fluoritas asociadas a rocas carbonatadas y las más ricas en las fluoritas que se encuentran encajadas en rocas ígneas y metamórficas. Se comprobó que las arcillas hidrotermales derivan de la alteración de las rocas volcánicas y que la barita presenta un patrón típico de barita continental.

Predomina la fluorita con relaciones $\mathrm{La} / \mathrm{Lu}$ mayores a 1 que se relaciona con la presencia de fluidos de naturaleza ácida. La fluorita asociada a sulfuros de Taxco está empobrecida en lantánidos ligeros respecto a la fluorita no asociada a sulfuros. Las muestras del distrito de Zacualpan (asociadas a rocas metamórficas y con trazas de sulfuro) presentan patrones más horizontales de lantánidos e incluso enriquecimiento en lantánidos pesados respecto a los lantánidos ligeros.

Las fluoritas claras o transparentes están, en general, menos enriquecidas en lantánidos que las fluoritas oscuras y/o violáceas. Sin embargo, no se puede establecer una correlación univoca entre el color y el contenido de estos elementos.

Los valores de la relación La/Ho nos confirman la presencia de procesos postdeposicionales. Geoquímicamente puede establecerse una relación entre la fluorita y fluidos que derivan de fundidos silicatados así como una tendencia marcada hacia el campo que se considera característico de la fluorita hidrotermal.

Predomina la fluorita con anomalía negativa de Eu (condiciones oxidantes), pero existen algunos episodios iniciales de fluorita con anomalía positiva de $\mathrm{Eu}$ (condiciones reductoras y fluido de mayor temperatura).

Para el distrito minero de Taxco, se diferencian dos grupos de fluoritas denominados F1 y F2. Dentro de cada grupo existen varias generaciones. F1 son fluoritas asociadas al reemplazo de carbonato, empobrecidas en lantánidos y con texturas primarias muy variables. F2 son fluoritas más tardías, en general de color más claro, no asociadas al reemplazo del carbonato y con texturas típicamente secundarias, con mayor enriquecimiento en REE y anomalías negativas de europio. 
(a)
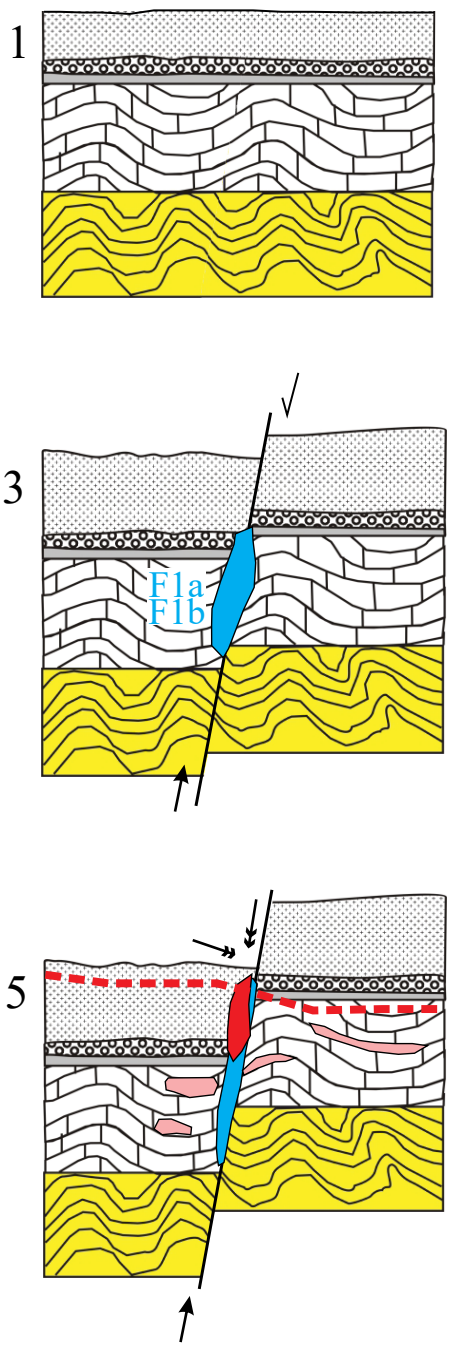
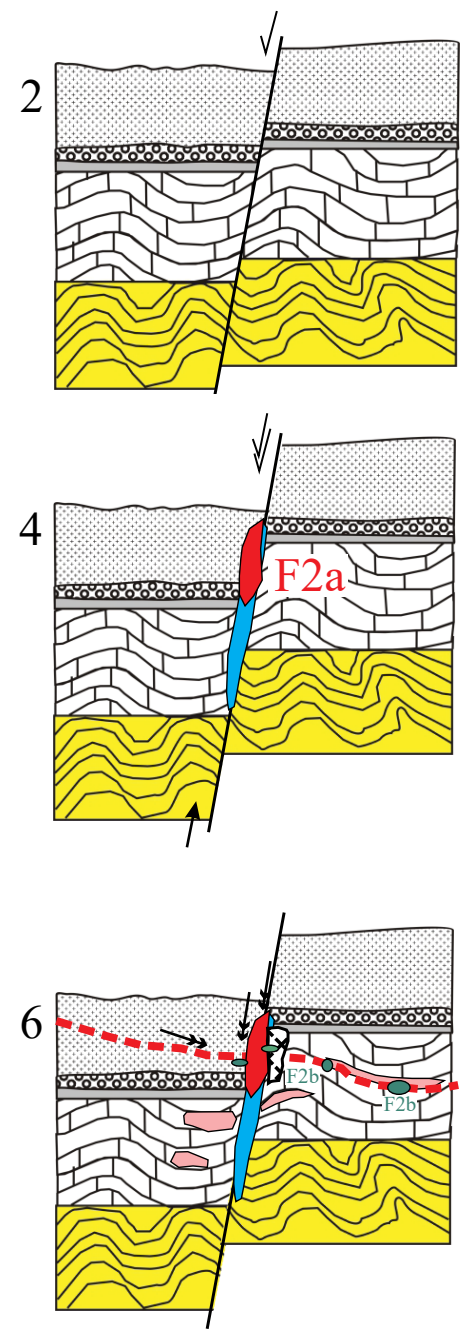

Rocas volcánicas del Cenozoico

PO Materiales detríticos continentales (Fm Balsas)

Materiales detríticos marinos (Fm Mexcala)

Carbonatos de plataforma

(Fm Morelos)

Rocas metamórficas en

7 fácies esquisto verde (Esquisto Taxco)

Fluorita temprana asociada al reemplazo de carbonato (F1a, F1b)

Fluorita tardía (F2a)

Fluorita tardía (F2b) asociada a la remobilización de F1

Cavidades formadas por disolución del carbonato

(1) Falla normal

- Erosión

(๖) Circulación de fluidos meteóricos

(1) Circulación de fluidos hidrotermales

Hundimiento cárstico y formación de brechas de colapso

(b))

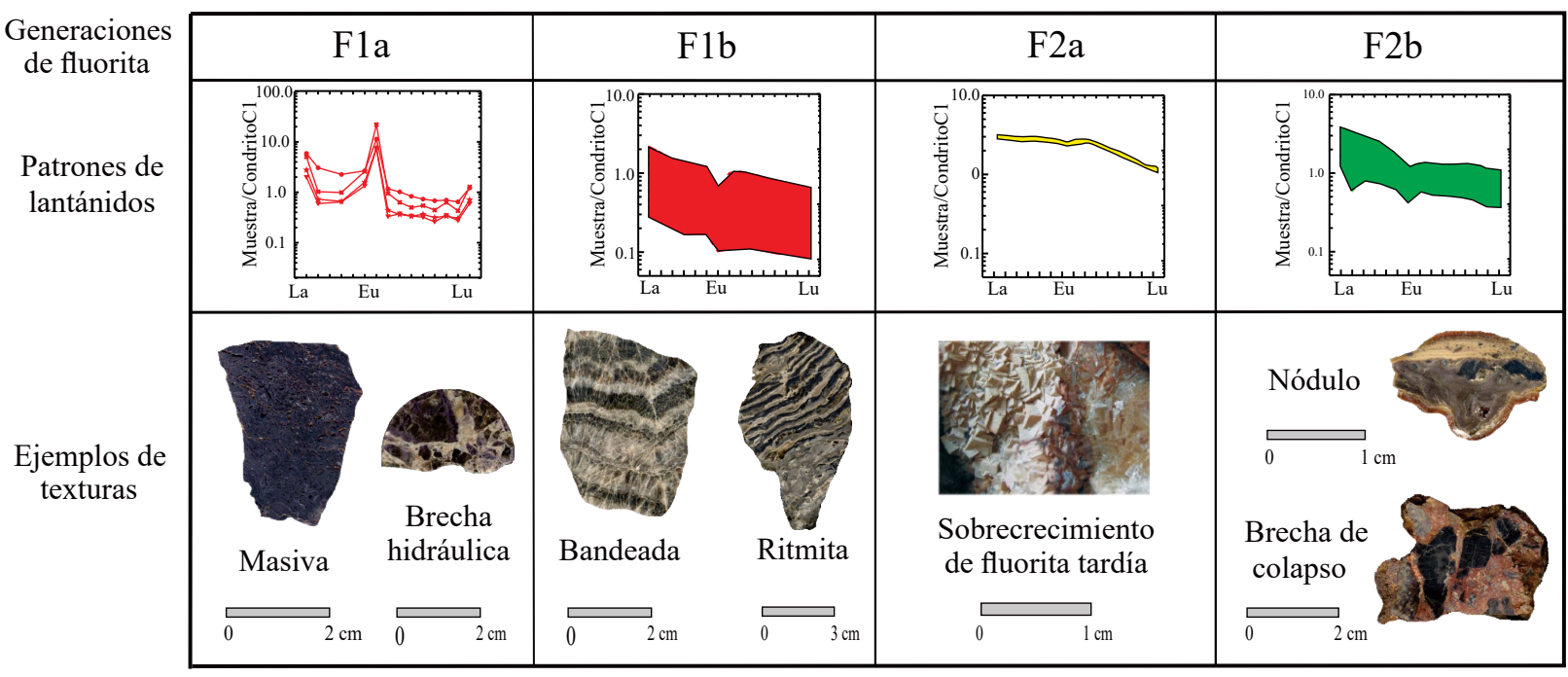

Figura 8. Modelo conceptual (a) y evolutivo (b) de las diferentes generaciones de fluorita de la mina La Azul, Taxco. 


\section{AGRADECIMIENTOS}

Los autores agradecen a Juan Pablo Bernal Uruchurtu, Patricio Montecinos Muñoz y dos revisores anónimos los comentarios al documento inicial, que fueron muy valiosos para mejorar el trabajo. Así mismo, se agradece el apoyo editorial de Ángel Nieto Samaniego, Peter Schaaf y Luigi Solari. Este trabajo fue parcialmente financiado por el Programa de Apoyo a Proyectos de Investigación e Innovación Tecnológica (PAPIIT) de la UNAM, IN111917.

\section{SUPLEMENTO ELECTRÓNICO}

La Tabla A1, puede ser descargada del sitio:http://satori.geociencias.unam.mx, en la tabla de contenido de este volumen.

\section{REFERENCIAS}

Alaniz-Álvarez, S.A., Nieto-Samaniego, A.F., Morán-Zenteno, D., Alba-Aldave, L. 2002, Rhyolitic volcanism in extension zone associated with strike-slip tectonics in the Taxco region, southern Mexico: Journal of Volcanology and Geothermal Research, 118(1), 1-14.

Allen, R.D., 1952, Variations in chemical and physical properties of fluorite: American Mineralogist, 37, 910-930.

Bau, M., 1996, Controls on the fractionation of isovalent trace elements in magmatic and aqueous systems: evidence from $\mathrm{Y} / \mathrm{Ho}, \mathrm{Zr} / \mathrm{Hf}$, and lanthanide tetrad effect: Contributions to Mineralogy and Petrology, $123,323-333$.

Bau, M., Dulski, P., 1995, Comparative study of yttrium and rare-earth element behaviours in fluorine-rich hydrothermal fluids: Contributions to Mineralogy and Petrology, 119, 213-223.

Bau, M., Romer, R.L., Lüders, V.Y., Dulski, P., 2003, Tracing element sources of hydrothermal mineral deposits: $\mathrm{REE}$ and $\mathrm{Y}$ distribution and $\mathrm{Sr}-\mathrm{Nd}-\mathrm{Pb}$ isotopes in fluorite from MVT deposits in the Pennine Orefield, England: Mineralium Deposita, 38, 992-1008.

Barrat, J.A., Zanda, B., Moynier, F., Bollinger, C., Liorzou, C., Bayon, G., 2012, Geochemistry of CI chondrites: Major and trace elements, and $\mathrm{Cu}$ and Zn Isotopes: Geochimica et Cosmochimica Acta, Elsevier, 83, 79-92.

Bosze, S., Rakovan, J., 2002, Surface structure controlled sectorial zoning of the rare earth elements in fluorite from Long Lake, New York, and Bingham, New Mexico, USA: Geochimica et Cosmochimica Acta, 66, 997-1009.

Campa-Uranga, M.F., Torres de León, R., Iriondo, A., Premo, W.R. 2012, Caracterización geológica de los ensambles metamórficos de Taxco y Taxco el Viejo, Guerrero, México: Boletín de la Sociedad Geológica Mexicana, 64(3), 369-385.

Chatagnon, B., Galland, D., 1982, R.P.E. de la fluorite naturelle: inventaire des ions lanthanides et autres impuretés paramagnétiques: Bulletin Minéralogie, 105, 37-42.

Constantopoulos, J., 1988, Fluid inclusions and REE geochemistry of fluorite from S-Central Idaho: Economic Geology, 83, 626-636.

Ehya, F., 2012, Variation of mineralizing fluids and fractionation of REE during the emplacement of the vein-type fluorite deposit at Bozijan, Markazi Province, Iran: Journal of Geochemical Exploration, 112, 93-106.

Ekambaram, V., Brookins, D.G., Rosenburg, P.E., Emanuel, K.M., 1986, Rareearth element geochemistry of fluorite-carbonate deposits in Western Montana, U.S.A.: Chemical Geology, 54, 319-331.

Eppinger, R.G., Closs, L.G., 1990, Variation of trace elements and REE in Fluorite: a possible tool for exploration: Economic Geology, 85, 1896-1907.

Fanlo, I., Touray, J.C., Subias I., Fernández-Nieto, 1998, Geochemical patterns of a sheared fluorite vein, Parzan Spanish Central Pyrenees: Mineralium Deposita, 33(6), 620-632.

Fleischer, M., 1969, The lanthanide elements in fluorite: Indian Mineralogy, 10, 36-39.

Gagnon, J.E., Samson, I.M., Fryer, B.J., Williams-Jones, E., 2003, Compositional heterogeneity in fluorite and the genesis of fluorite deposits: insights from LA-ICP-MS analysis: The Canadian Mineralogist, 41, 365-382.
Grappin, C., Treuil, M., 1979, Le Spèctre des Terres Rares de la fluorine en tant que marqueur des propriétés du milieu de dépôt et des interactions entre solutions minéralisantes et roches sources: Exemple pris dans le district de la Marche Occidentale (France): Mineralium Deposita, 14, 297-309.

Graupner,T., Mühlbach, C., Schwarz-Schampera, U., Henjes-Kunst,F., Melcher, F., Terblanche, H., 2015, Mineralogy of high-field-strength elements (Y, $\mathrm{Nb}, \mathrm{REE}$ ) in the world-class Vergenoeg fluorite deposit, South Africa: Ore Geology Reviews, 64, 583-601.

Haas, J.R., Shock, E.L., Sarrani, D.V., 1993, Prediction of high-temperature stability constants for aqueous compounds of the rare-earth elements: Geological Society of America, Annual Meeting, Boston, Massachusetts, Abstract with programs, 25(6), 437.

Hill, G.T., Campbell, A.R., Kyle P.R., 2000, Geochemistry of southwestern New Mexico fluorite occurrences implications for precious metals exploration in fluorite bearing systems: Journal of Geochemical Exploration, 68(1-2), $1-20$.

Irving, A.J., 1978, A review of experimental studies of crystal/liquid trace element partitioning: Geochimica and Cosmochimica Acta, 42, 743-770.

Jebrak, M., Smejkal, V., Albert D., 1985, Rare earth and isotopic geochemistry of the fluorite-barite vein deposits from the western Rouergue District (France): Economic Geology, 80, 2030-2034.

Krupenin, M.T., Moeller, P., Dulski, P., 1999, Rare earth and other elements in fluorite and in the lower Riphean Host Dolomite of the Southern Urals: Geochemistry International, 11, 1165-1177.

Lottermoser, B.G.,1992, REE and Hydrothermal ore formation processes: Ore Geology Reviews, 7, 25-41.

Makin, S.A., Simandl, G.J., Marshall, D., 2014, Fluorite and its potential as an indicator mineral for carbonatite related rare earth element deposits: Geological Fieldwork 2013, British Columbia Ministry of Energy and Mines, British Columbia Geological Survey, Paper 2014-1, 207-212.

Marchand, L., Joseph, D., Touray J.C.,1976, Critères d'analyse géochimique des gisements de fluorine basés sur 1'étude de la distribution des lanthanidesapplication au gite de Maine (71-Cordesse, France): Mineralium Deposita $11,357-379$.

Meary, A., Touray, J.C., Galland, D., Jebrak M., 1985, Interprétation de l'anomalie en europium des fluorines hydrothermales-données de la résonance paramagnétique électronique: application au gite de fluorine de Montroc (Tarn France): Chemical Geology, 48, 115-124.

McDonough, W.F., Sun, S.S., 1995, The Composition of the Earth: Chemical Geology, 120, 223-253.

Möller, P. Morteani, G.,1983, On the geochemical fractionation of rare earth elements during the formation of Ca-minerals and its application to problems of the genesis of ore deposits, en Augusthitis S.S. (ed.), The significance of trace elements in solving petrogenetic problems and controversies: Athens, Theophrastus Publications, 747-791.

Möller P., Parekh P.P., Schneider H.J., 1976, The application of Tb/Ca - Tb/La abundance ratios to problems of fluorspar genesis: Mineralium Deposita, $11,111-116$.

Monecke, T., Monecke, J., Mönch, W. Kempe, U., 2000, Mathematical analysis of rare earth element patterns of fluorites from the Ehrenfriedersdorf tin deposit, Germany: evidence for a hydrothermal mixing process of lanthanides from two different sources: Mineralogy and Petrology 70: 235. doi:10.1007/s007100070005.

Morgan, J.W., Wandless, A.,1980, Rare-earth element distribution in some hydrothermal minerals: evidence for crystallographic control: Geochimica et Cosmochimica Acta, 44,973-980.

Pi, T., 2005, Estudio mineralógico, geoquímico y geocronológico de los yacimientos de fluorita de Taxco (Guerrero) y Zacualpan (Edo. De México): Universidad Nacional Autónoma de México, tesis de doctorado, $256 \mathrm{pp}$.

Pi, T., Solé, J., Taran, Y., 2005a, Role of water-rock interaction in the formation of La Azul fluorite deposit, southern Mexico, en Wanty R.R., Seal II (eds.). Water-rock Interaction: London, Taylor and Francis Group, ISBN 90-5809 6416, vol 1, 299-302.

Pi T., Solé, J., Taran, Y., 2005b, (U-Th)/He dating of fluorite: application to the La Azul fluorspar deposit in the Taxco mining district, Mexico: Mineralium Deposita 39, (8), 976-982.

Rivera Carranza, E., de la Teja Segura, M.A, Miranda Huerta, A., Lemus Bustos, O., Motolínia García, O., León Ayala, V., Moctezuma Salgado M.,D., 
1998, Hoja Cuernavaca 90_E14-5, escala 1:250000: Servicio Geológico Mexicano, Pachuca, 1 mapa.

Ruiz, J., Kesler, S.E., Jones, L.M., Sutter, J.F., 1980, Geology and Geochemistry of the Las Cuevas fluorite deposit, San Luis Potosí, Mexico: Economic Geology 75, 1200-1209.

Sasmaz, A., Yavuz, F., 2007, REE geochemistry and fluid-inclusion studies of fluorite deposits from the Yaylagözü area (Yıldızeli-Sivas) in Central Turkey: Neues Jahrbuch für Mineralogie,183(2), 215-226.

Schwinn G., Markl G., 2005, REE systematics in hydrothermal fluorite: Chemical Geology 216, 225-248.

Strong, D.F., Fryer, B.J., Kerrich, R.,1984, Genesis of the St. Lawrence fluorspar deposits as indicated by fluid inclusion, rare earth element and isotopic data. A second issue devoted to Canadian mineral deposits: Economic Geology, 79(5), 1142-1158.

Subías, I., Fanlo, I., López- Ciriano, A., Ferrnández-Nieto, C., 1994, Geochemistry and genesis of fluorite mineralization of Spanish Western Pyrenees (Huesca Province), en Seltmann R., Kämpf H., Moeller, P. (eds), Metallogeny of collisional orogens: Prague, Czech Geological Survey, 423-429.

Soussi, F., Souissi, R., Dandurand, J.L., 2012, The Genesis of the Mississippi Valley-Type Fluorite Ore at Jebel Stah (Zaghouan District, North-Eastern Tunisia), Constrained by Thermal and Chemical Properties of Fluids and REE and Sr Isotope Geochemistry, en Panagiotaras, D. (ed.), Geochemistry - Earth's System Processes, InTech., 249-290.

Sverjensky, D.A., 1984, Europium redox equilibria in aqueous solution: Earth and Planetary Science Letters, 67, 70-78.

Trinkler, M., Monecke, T., Thomas, R., 2005, Constraints on the genesis of yellow fluorite in hydrothermal barite-fluorite veins of the Erzgebirge, Eastern Germany: Evidence from optical absorption spectroscopy, rare earth element data, and fluid inclusion investigations: Canadian Mineralogist, 43(3), 883-898.
Walker, J.B., Choppin, G.R., 1967, Thermodynamic parameters of fluoride complexes of the lanthanides: Advances in Chemistry, 71, 127-140.

William-Jones, A.E., Samson, I.M., Olivo, G.R., 2000, Genesis of hydrothermal fluorite-REE deposits in the Gallinas Mountains, New Mexico: Economic Geology, 95, 327-342.

Whittaker, E.J.W., Muntus, R., 1972, Ionic radii for use in geochemistry: Geochimica et Cosmochimica Acta, 34, 945-956.

Wood, S.A., 1990, The aqueous geochemistry of the rare-earth elements and yttrium. 2. Theoretical predictions of speciation in hydrothermal solutions to $350^{\circ} \mathrm{C}$ at saturation water vapor pressure: Chemical Geology, 88 (1-2), 99-125.

Xu, C., Taylor, R.N., Li, W., Kynicky, J., Chakhmouradian, A.R., Song, W., 2012, Comparison of fluorite geochemistry from REE deposits in the Panxi region and Bayan Obo, China: Journal of Asian Earth Sciences, 57, 76-89. (doi:10.1016/j.jseaes.2012.06.007).

Manuscrito recibido: diciembre 23, 2016

Manuscrito corregido recibido: agosto 17, 2017

Manuscrito aceptado: agosto 18, 2017 